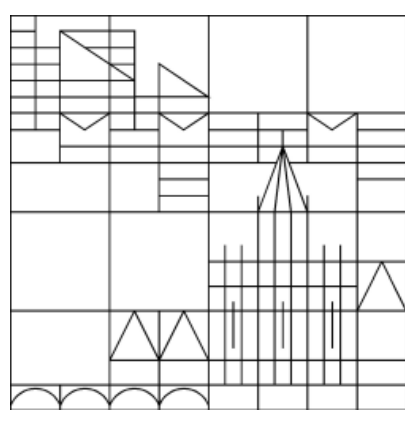

\title{
Trace-positive polynomials and the quartic tracial moment problem
}

\author{
Sabine Burgdorf \\ Igor Klep
}

Konstanzer Schriften in Mathematik

Nr. 269, Juni 2010

ISSN 1430-3558

Konstanzer Online-Publikations-System (KOPS)

URN: http://nbn-resolving.de/urn:nbn:de:bsz:352-opus-119770

URL: http://kops.ub.uni-konstanz.de/volltexte/2010/11977/ 



\title{
Trace-positive polynomials and the quartic tracial moment problem Polynômes avec une trace positive et le problème quartique des moments traciaux
}

\author{
Sabine Burgdorf ${ }^{\mathrm{a}, 1,3}$, Igor Klep $\mathrm{p}^{\mathrm{b}, 2,3}$ \\ ${ }^{a}$ Institut de Recherche Mathématique de Rennes, Université de Rennes 1, Campus de Beaulieu, 35042 Rennes cedex, France, \\ and Universität Konstanz, Fachbereich Mathematik und Statistik, 78457 Konstanz, Germany \\ ${ }^{b}$ Univerza $v$ Mariboru, Fakulteta za naravoslovje in matematiko, Koroška 160, 2000 Maribor, \\ and Univerza $v$ Ljubljani, Fakulteta za matematiko in fiziko, Jadranska 19, 1000 Ljubljana, Slovenia
}

\begin{abstract}
The tracial analog of Hilbert's classical result on positive binary quartics is presented: a trace-positive bivariate noncommutative polynomial of degree at most four is a sum of hermitian squares and commutators. This is applied via duality to investigate the truncated tracial moment problem: a sequence of real numbers indexed by words of degree four in two noncommuting variables with values invariant under cyclic permutations of the indexes, can be represented with tracial moments of matrices if the corresponding moment matrix is positive definite. Understanding trace-positive polynomials and the tracial moment problem is one of the approaches to Connes' embedding conjecture.
\end{abstract}

\section{Résumé}

Nous présentons l'analogue tracial du résultat classique de Hilbert sur les quartiques positives: un polynôme de degré quatre en deux variables non commutatives ayant une trace positive est une somme de carrés hermitiens et de commutateurs. Ceci est appliqué par dualité à l'étude du problème tronqué des moments traciaux: une suite de nombres réels indexée par des mots de degré quatre en deux variables non commutatives, ayant des valeurs invariantes par permutations circulaires des indices, peut être représentée par des moments traciaux, si la matrice des moments est définie positive.

Keywords: noncommutative polynomial, trace, sum of hermitian squares, (truncated) moment problem, free positivity

2010 MSC: Primary 11E25, 44A60; Secondary 08B20, 15A45, 13J30

\section{Version abrégée en français}

Soit $\mathbb{R}\langle X, Y\rangle$ l'anneau des polynômes en des variables $X, Y$ non commutatives muni de l'involution $p \mapsto p^{*}$ avec $X^{*}=X, Y^{*}=Y$ et $a^{*}=a$ pour $a \in \mathbb{R}$. Des élements de la forme $g_{i}^{*} g_{i}$ sont des sommes de carrés hermitiens. Un polynôme $f \in \mathbb{R}\langle X, Y\rangle$ a une trace positive $\operatorname{si} \operatorname{tr}(f(A, B)) \geqslant 0$ pour toutes matrices symétriques $A, B \in \mathbb{S R}^{n \times n}, n \in \mathbb{N}$. Evidemment, une somme de carrés hermitiens a une trace positive.

Comme la trace est invariante par permutation circulaire des matrices, c'est-à-dire, $\operatorname{tr}(A B-B A)=0$ pour toutes matrices $A, B \in \mathbb{R}^{n \times n}$, nous pouvons ajouter à $f$ des commutateurs $p q-q p$ de polynômes $p, q \in$ $\mathbb{R}\langle X, Y\rangle$ sans changer sa trace. On note l'ensemble des sommes de carrés hermitiens et de commutateurs par $\Theta^{2}$. Tous les $f \in \Theta^{2}$ ont une trace positive, mais, en général, on n'a pas la réciproque. Par exemple, $M_{\mathrm{nc}}=X Y^{4} X+Y X^{4} Y-3 X Y^{2} X+1 \in \mathbb{R}\langle X, Y\rangle$, une version non commutative du polynôme de Motzkin,

\footnotetext{
Email addresses: sabine.burgdorf@univ-rennes1.fr (Sabine Burgdorf), igor.klep@fmf.uni-lj.si (Igor Klep)

${ }^{1}$ The first author was partially supported by the Zukunftskolleg Konstanz.

${ }^{2}$ The second author was partially supported by the Slovenian Research Agency (program no. P-0222).

${ }^{3}$ Both authors were supported by the French-Slovene partnership project Proteus 20208ZM. We thank Ronan Quarez and Markus Schweighofer for freely sharing their knowledge and skills.
} 
a une trace positive mais n'est pas un élément de $\Theta^{2}[\mathrm{KS}$. Cependant, nous présentons la démonstration de la version traciale du résultat classique de Hilbert [Hil] sur les quartiques positives en deux variables.

Théorème 1. Soit $f \in \mathbb{R}\langle X, Y\rangle$ de degré quatre. Alors les assertions suivantes sont équivalentes:

(i) $f$ a une trace positive;

(ii) $f \in \Theta^{2}$.

Ce résultat comprend les cas faciles d'un polynôme univarié $f$ et d'un polynôme bivarié quadratique.

Ce théorème peut être appliqué par dualité à l'étude du problème tronqué des moments traciaux; un analogue du problème des moments d'Analyse fonctionnelle dans notre contexte, qui a été introduit dans [BK]. Une suite $y=\left(y_{w}\right)_{\leqslant 2 k}$ traciale et tronquée est une suite de nombres réels indexée par des mots en deux variables non commutatives de degré $\leqslant 2 k$, dont les valeurs sont invariantes par l'involution $*$ et par permutation circulaire des indices. Par exemple, une suite donnée par

$$
y_{w}:=\int \operatorname{tr}(w(A, B)) \mathrm{d} \mu(A, B)
$$

pour une mesure de probabilité $\mu$ sur $\left(\mathbb{S R}^{n \times n}\right)^{2}$ et pour un $n \in \mathbb{N}$, est une suite traciale.

Nous nous intéressons au problème réciproque (le problème tronqué des moments traciaux): Pour quelles suites $y=\left(y_{w}\right)_{\leqslant k}$, où $w$ est borné $\operatorname{par} \operatorname{deg} w \leqslant k$ pour un $k \in \mathbb{N}$, existe-t-il un $n \in \mathbb{N}$, une mesure $\mu$ de probabilité sur $\left(\mathbb{S R}^{n \times n}\right)^{2}$ tels qu'on ait une réprésentation comme en (1)? Dans ce cas, il $y$ a une représentation traciale et $y$ est dit une suite tronquée des moments traciaux. Plusieurs résultats classiques sont transférables aux suites tronquées et traciales, par exemple, le théorème de Bayer-Teichmann [BT, Théorème 2], voir [BCKP, Theorem 4.5] pour la version traciale, ou les résultats de Curto et Fialkow [CF] sur le problème des moments plats, cf. [BK, Corollary 3.19].

Les suites traciales décrivent des formes linéaires traciales. La forme bilinéaire induite par $y$ est caractérisée par sa matrice $M_{k}(y)$ des moments traciaux, soit, la matrice $M_{k}(y)=\left[y_{u} *_{v}\right]_{u, v}$, indexée par des mots $u, v$ avec $\operatorname{deg} u, \operatorname{deg} v \leqslant k$. Le résultat classique de Hilbert a été utilisé par Fialkow et Nie pour résoudre le problème tronqué des moments bivariés quartiques [FN], Théorème 3.3] et nous en démontrons la version traciale.

Corollaire 2. Soit $y=\left(y_{w}\right)_{\leqslant 4}$ une suite traciale et tronquée telle que sa matrice des moments soit définie positive. Alors, y est une suite de moments traciaux.

\section{Introduction}

Starting from Helton's influential paper [Hel], in which he proved that a noncommutative polynomial is a sum of hermitian squares if and only if its values in matrices of any size are positive semidefinite, free positivity, the emerging area dealing with noncommutative inequalities, mostly in a free algebra, is expanding rapidly. Considering polynomials with positive trace, Schweighofer and the second author in KS observed that Connes embedding conjecture [Con, Section V, pp. 105-107] on type $\mathrm{II}_{1}$ von Neumann algebras is equivalent to a problem of describing polynomials all of whose values at tuples of matrices (of norm at most 1) have positive trace (see $[\mathrm{KS}$, Theorem 1.6] for a precise statement). This result is the motivation for the present work. Here we investigate trace-positive polynomials of low degree and establish a tracial analog of the classical result of Hilbert [Hil] on positive (homogeneous) ternary quartics: a trace-positive bivariate noncommutative polynomial of degree at most four is a sum of hermitian squares and commutators.

\section{Notation}

Let $\langle X, Y\rangle$ denote the free monoid generated by $X$ and $Y$, i.e., $\langle X, Y\rangle$ consists of words in the noncommuting letters $X$ and $Y$. We consider the free algebra $\mathbb{R}\langle X, Y\rangle$ of polynomials in the noncommuting variables $X, Y$ with coefficients in $\mathbb{R}$. Endow $\mathbb{R}\langle X, Y\rangle$ with the involution $p \mapsto p^{*}$ fixing $\mathbb{R} \cup\{X, Y\}$ pointwise. Hence 
for each word $w \in\langle X, Y\rangle, w^{*}$ is its reverse. The length of the longest word in a polynomial $f \in \mathbb{R}\langle X, Y\rangle$ is the degree of $f$ and is denoted by $\operatorname{deg} f$. We write $\mathbb{R}\langle X, Y\rangle_{\leqslant k}$ for the set of all polynomials of degree $\leqslant k$.

An element of the form $g^{*} g$ for some $g \in \mathbb{R}\langle X, Y\rangle$ is called a hermitian square and we denote the set of all sums of hermitian squares by $\Sigma^{2}:=\left\{\sum_{i=1}^{N} g_{i}^{*} g_{i} \mid N \in \mathbb{N}, g_{i} \in \mathbb{R}\langle X, Y\rangle\right\}$. A polynomial $f \in \mathbb{R}\langle X, Y\rangle$ is matrix-positive if $f(A, B)$ is positive semidefinite for all symmetric matrices $A, B \in \mathbb{S R}^{n \times n}, n \in \mathbb{N}$. Helton Hel (and, independently McCullough $[\mathrm{McC}$ ) proved that $f \in \mathbb{R}\langle X, Y\rangle$ is matrix-positive if and only if $f \in \Sigma^{2}$ by solving a noncommutative moment problem.

Definition 2.1. A polynomial $f \in \mathbb{R}\langle X, Y\rangle$ is trace-positive if $\operatorname{tr}(f(A, B)) \geqslant 0$ for all $A, B \in \mathbb{S R}^{n \times n}, n \in \mathbb{N}$.

Trace-positive polynomials are intimately connected to deep open problems from e.g. operator algebras (Connes' embedding conjecture [Con, Section V, pp. 105-107]; see [KS, Theorem 1.6]), so a good understanding of this set is needed. The invariance of the trace under cyclic permutations motivates the following definition of cyclic equivalence [KS, p. 1817].

Definition 2.2. $f, g \in \mathbb{R}\langle X, Y\rangle$ are cyclically equivalent $(f \stackrel{\text { cyc }}{\sim} g)$ if $f-g$ is a sum of commutators.

Two words $v, w \in\langle X, Y\rangle$ are cyclically equivalent if and only if $w$ is a cyclic permutation of $v$. Equivalently: there exist $u_{1}, u_{2} \in\langle X, Y\rangle$ such that $v=u_{1} u_{2}$ and $w=u_{2} u_{1}$. Less obvious is that symmetric $f, g \in \mathbb{R}\langle X, Y\rangle$ are cyclically equivalent if and only if $\operatorname{tr}(f(A, B))=\operatorname{tr}(g(A, B))$ for all $A, B \in \mathbb{S R}^{n \times n}, n \in \mathbb{N}$ [KS, Theorem 2.1].

Definition 2.3. Let $w \in\langle X, Y\rangle$. The canonical representative $[w]$ of $w$ is the Lyndon word associated to $w$, i.e., the smallest word with respect to the lexicographic order which is cyclically equivalent to $w$. For $f=\sum_{w} a_{w} w \in \mathbb{R}\langle X, Y\rangle$, the canonical representative of $f$ is $[f]:=\sum_{[w]} a_{[w]}[w] \in \mathbb{R}\langle X, Y\rangle$. That is, [f] contains only canonical representatives of words from $f$ with coefficients $a_{[w]}:=\sum_{u}{ }_{\sim}{ }_{w}{ }_{w} a_{u}$.

Definition 2.4. The set of all polynomials cyclically equivalent to a sum of hermitian squares is

$$
\Theta^{2}:=\left\{f \in \mathbb{R}\langle X, Y\rangle \mid f \stackrel{\text { cyc }}{\sim} \sum_{i=1}^{N} g_{i}^{*} g_{i} \text { for some } N \in \mathbb{N}, g_{i} \in \mathbb{R}\langle X, Y\rangle\right\} .
$$

Obviously, all $f \in \Theta^{2}$ are trace-positive. However, in contrast to Helton's sum of squares theorem mentioned above, the following noncommutative version of the well-known Motzkin polynomial shows that a trace-positive polynomial need not be a member of $\Theta^{2}[\mathrm{KS}$. The Motzkin polynomial is a famous positive (commutative) polynomial that is not a sum of squares. For other examples see e.g. CLR, Rez, Mar, PD.

Example 2.5. Let

$$
M_{\mathrm{nc}}=X Y^{4} X+Y X^{4} Y-3 X Y^{2} X+1 \in \mathbb{R}\langle X, Y\rangle .
$$

Then $M_{\mathrm{nc}} \notin \Theta^{2}$ since the commutative Motzkin polynomial is not a sum of squares [Mar, p. 5]. The fact that $M_{\mathrm{nc}}$ is trace-positive is not obvious and has been shown by Schweighofer and the second author $\mathrm{KS}$, Example 4.4] using Putinar's Positivstellensatz [Put]. We point out that a more elementary proof using the spectral theorem has been given by Ken Dykema in 2008 (private communication).

\section{Main results}

In this section we present our main results. The first subsection studies bivariate trace-positive polynomials of low degree, while Subsection 3.2 applies these results to the dual problem, i.e., to the truncated tracial moment problem [BK].

\subsection{Trace-positive polynomials}

It is easy to see that a univariate polynomial $f \in \mathbb{R}\langle X\rangle$ is (trace-)positive if and only if $f \in \sum \mathbb{R}\langle X\rangle^{2}$. Similarly, a quadratic polynomial $f \in \mathbb{R}\langle X, Y\rangle \leqslant 2$ is trace-positive if and only if $f \in \Theta^{2}$. On the other hand, trace-positive polynomials of degree $\geqslant 6$ need not be in $\Theta^{2}$; see Example 2.5. The degree gap is bridged by the following theorem: 
Theorem 3.1. For $f \in \mathbb{R}\langle X, Y\rangle_{\leqslant 4}$, the following statements are equivalent:

(i) $f$ is trace-positive;

(ii) $f$ is trace-positive on $\left(\mathbb{S R}^{2 \times 2}\right)^{2}$;

(iii) $f$ is cyclically equivalent to a sum of four hermitian squares;

(iv) $f \in \Theta^{2}$.

Loosely speaking, the idea of the proof is as follows. If the coefficient of a trace-positive $f \in \mathbb{R}\langle X, Y\rangle \leqslant 4$ belonging to $X^{2} Y^{2}$ is at least as big as the one belonging to $X Y X Y$, then the membership certificate $f \in \Theta^{2}$ can be explicitly constructed from a sum of squares certificate for the commutative collapse of $f$ (Lemma 3.2). In the remaining case a clever linear transformation is applied to reduce the problem to one we can handle as above (Lemmas 3.4 and 3.3 .

We replace $f$ by its canonical representative $[f]$ and denote the coefficients $a_{[w]}$ of $f=[f]$ by $a_{w}$. Let $\breve{f}$ be the commutative collapse of $f$, i.e., $\breve{f}$ is obtained from $f$ by replacing the noncommuting variables $X, Y$ with commuting variables $x, y$.

Lemma 3.2. Let $f \in \mathbb{R}\langle X, Y\rangle_{\leqslant 4}$. If $\check{f} \geqslant 0$ on $\mathbb{R}^{2}$ and $a_{X^{2} Y^{2}} \geqslant a_{X Y X Y}$, then $f \in \Theta^{2}$. In fact, $f$ is cyclically equivalent to a sum of four hermitian squares.

Proof. Since $\breve{f} \geqslant 0$ on $\mathbb{R}^{2}$, by the classical result of Hilbert $\underline{\text { Hil }}$, there exist $\breve{g}_{1}, \breve{g}_{2}, \breve{g}_{3} \in \mathbb{R}[x, y]^{2}$ such that $\check{f}=\sum_{i=1}^{3} \check{g}_{i}^{2}$. Each $\check{g}_{i}$ can be lifted to

$$
g_{i}:=c_{0}^{(i)}+c_{1}^{(i)} X+c_{2}^{(i)} Y+c_{3}^{(i)} \frac{X Y+X Y}{2}+c_{4}^{(i)} X^{2}+c_{5}^{(i)} Y^{2} \in \mathbb{R}\langle X, Y\rangle .
$$

One easily verifies that

$$
\sum_{i=1}^{3} g_{i}^{*} g_{i} \stackrel{\text { cyc }}{\sim} f-\left(\left(a_{X^{2} Y^{2}}-\frac{a_{X^{2} Y^{2}}+a_{X Y X Y}}{2}\right) X^{2} Y^{2}+\left(a_{X Y X Y}-\frac{a_{X^{2} Y^{2}}+a_{X Y X Y}}{2}\right) X Y X Y\right) .
$$

Taking $g_{4}:=\sqrt{\frac{a_{X^{2} Y^{2}}-a_{X Y X Y}}{2}}(X Y-Y X) \in \mathbb{R}\langle X, Y\rangle$, we obtain $f \stackrel{\text { cyc }}{\sim} \sum_{i=1}^{4} g_{i}^{*} g_{i} \in \Theta^{2}$.

As a consequence of Lemma 3.2 we derive a criterion for biquadratic polynomials to be members of $\Theta^{2}$. A polynomial $f \in \mathbb{R}\langle X, Y\rangle$ is called biquadratic if $\operatorname{deg}_{X} f \leqslant 2$ and $\operatorname{deg}_{Y} f \leqslant 2$.

Lemma 3.3. Let $f \in \mathbb{R}\langle X, Y\rangle \leqslant 4$ be biquadratic. Then $f \in \Theta^{2}$ if and only if $f$ is trace-positive on $\left(\mathbb{S R}^{2 \times 2}\right)^{2}$.

Proof. Obviously, $f \in \Theta^{2}$ implies $f$ is trace-positive. Conversely, if $f$ is trace-positive on $\left(\mathbb{S R}^{2 \times 2}\right)^{2}$, then by considering

$$
A_{x}:=x\left[\begin{array}{ll}
0 & 1 \\
1 & 0
\end{array}\right] \text { and } B_{y}:=y\left[\begin{array}{cc}
1 & 0 \\
0 & -1
\end{array}\right]
$$

we obtain that the (commutative) polynomial

$$
p(x, y):=\operatorname{tr}\left(f\left(A_{x}, B_{y}\right)\right)=a_{1}+a_{X^{2}} x^{2}+a_{Y^{2}} y^{2}+\left(a_{X^{2} Y^{2}}-a_{X Y X Y}\right) x^{2} y^{2} \in \mathbb{R}[x, y]
$$

is nonnegative on $\mathbb{R}^{2}$. This implies $a_{X^{2} Y^{2}}-a_{X Y X Y} \geqslant 0$. Since $\breve{f} \geqslant 0$ on $\mathbb{R}^{2}$, Lemma 3.2 yields $f \in \Theta^{2}$.

Lemma 3.4. If $f \in \mathbb{R}\langle X, Y\rangle \leqslant 4$ is trace-positive on $\left(\mathbb{S R}^{2 \times 2}\right)^{2}$, then there exists $G \in \mathrm{GL}_{2}(\mathbb{R})$, such that the coefficients $b_{w}$ of the canonical representative of $f\left(G\left[\begin{array}{c}X \\ Y\end{array}\right]\right)$ satisfy $b_{X^{2} Y^{2}} \geqslant b_{X Y X Y}$. 
Proof. Let $a_{X^{2} Y^{2}}<a_{X Y X Y}$. Then at least one of the coefficients of $X^{4}$ and $Y^{4}$ is nonzero. Otherwise $f$ is biquadratic since $\breve{f}$ is nonnegative and thus the words $X^{3} Y$ and $X Y^{3}$ cannot occur. As we have seen in the proof of Lemma 3.3 , the trace-positivity then implies $a_{X Y X Y} \geqslant a_{X^{2} Y^{2}}$. Without loss of generality, let $f$ contain $X^{4}$. Then $a_{X^{4}}>0$ since $\check{f}$ is nonnegative on $\mathbb{R}^{2}$. We set $G:=\left[\begin{array}{cc}1 & s \\ 0 & -1\end{array}\right] \in G_{2}(\mathbb{R})$ for some $s \geqslant \frac{1}{2 a_{X^{4}}}\left(a_{X^{2} Y}+\sqrt{a_{X^{2} Y}^{2}+4 a_{X^{4}}\left(a_{X Y X Y}-a_{X^{2} Y^{2}}\right)}\right)$. Then

$$
b_{X^{2} Y^{2}}=4 s^{2} a_{X^{4}}-2 s a_{X^{3} Y}+a_{X^{2} Y^{2}} \geqslant 2 s^{2} a_{X^{4}}-s a_{X^{3} Y}+a_{X Y X Y}=b_{X Y X Y},
$$

as desired.

Proof of Theorem 3.1. The implications (i) $\Rightarrow($ ii), (iii) $\Rightarrow$ (iv) and (iv) $\Rightarrow($ i) are obvious. Suppose $f$ is trace-positive on $\left(\mathbb{S R}^{2 \times 2}\right)^{2}$. If $a_{X Y X Y}>a_{X^{2} Y^{2}}$, then we apply Lemma 3.4 and obtain a trace-positive polynomial $g \in \mathbb{R}\langle X, Y\rangle$ that satisfies the assumptions of Lemma 3.2. Hence (iii) holds for $g$ and thus also for $f$. If $a_{X Y X Y} \leqslant a_{X^{2} Y^{2}}$, then (iii) holds by Lemma 3.2 .

Remark 3.5. The bound of four hermitian squares in Theorem 3.1 is sharp. Indeed, the polynomial $f=1+\frac{1}{2} X^{2}+X^{4}+Y^{4}+2 X Y X Y$ is cyclically equivalent to a sum of four but not three hermitian squares.

A nice modern treatment of Hilbert's ingenious proof Hil of his positive ternary quartics theorem is given in PRSS, $\mathrm{PR}$. A more elementary and functional-analytic proof is given in CLR; however this proof does not give the sharp bound on the number of squares needed.

\subsection{The truncated tracial moment problem}

The moment problem is a classical question in functional analysis [Akh, $\mathrm{KN}, \mathrm{ST}$, and its finite variant, the so-called truncated moment problem, has been championed by Curto and Fialkow [CF. In the noncommutative setting, the free moment problem has been solved by McCullough [McC]; see also Helton [Hel]. In BK] we introduced and studied the (truncated) tracial moment problem. Before stating how our Theorem 3.1 bears on this, we briefly review the tracial moment problem specialized to two variables.

Definition 3.6. A sequence of real numbers $\left(y_{w}\right)$ indexed by words $w \in\langle X, Y\rangle$ satisfying

$$
y_{w}=y_{u} \text { whenever } w \stackrel{\text { cyc }}{\sim} u, \quad y_{w}=y_{w} * \text { for all } w,
$$

and $y_{\varnothing}=1$, is called a (normalized) tracial sequence.

Example 3.7. Given $n \in \mathbb{N}$ and a positive probability measure $\mu$ on $\left(\mathbb{S R}^{n \times n}\right)^{2}$, the sequence given by

$$
y_{w}:=\int \operatorname{tr}(w(A, B)) \mathrm{d} \mu(A, B)
$$

is a tracial sequence since the traces of cyclically equivalent words coincide.

We are interested in the truncated version of the converse of this example (the truncated tracial moment problem): For which sequences $y=\left(y_{w}\right)_{\leqslant k}$, where $w$ is constrained by $\operatorname{deg} w \leqslant k$ for some $k \in \mathbb{N}$, do there exist $n \in \mathbb{N}$, a positive probability measure $\mu$ on $\left(\mathbb{S R}^{n \times n}\right)^{2}$ such that $(3)$ holds? We then say that $y$ has a tracial moment representation and call it a truncated tracial moment sequence.

In fact, this is a seemingly more general version using integrals over Borel measures as opposed to finitely atomic measures as is done in [BK]. However, in the truncated case both versions are equivalent by our tracial version of the Bayer-Teichmann theorem established in BCKP, Theorem 4.5].

The tracial moment matrix $M_{k}(y)$ of a truncated tracial sequence $y=\left(y_{w}\right)_{\leqslant 2 k}$ is $M_{k}(y)=\left[y_{u} * v\right]_{u, v}$, a matrix indexed by words $u, v$ with $\operatorname{deg} u, \operatorname{deg} v \leqslant k$. If $y$ is a truncated tracial moment sequence, then $M_{k}(y)$ is positive semidefinite. The converse is false, cf. [BK, Example 3.5]. Even if $M_{k}(y)$ is positive definite, $y$ need not have a tracial moment representation [BK, Example 4.9]. 
It is easy to see that a truncated tracial sequence $y=\left(y_{w}\right)_{\leqslant 2}$ of degree 2 is a tracial moment sequence if and only if $M_{1}(y)$ is positive semidefinite. Similarly, a truncated moment sequence $y=\left(y_{w}\right)_{\leqslant k}$ in one variable with positive definite moment matrix is a tracial moment sequence.

In general, for the truncated moment problem flatness governs the existence of truncated tracial moment sequences, resembling the situation in the classical moment problem. Recall: a (symmetric) extension $\tilde{A}=\left[\begin{array}{cc}A & B \\ B^{*} & C\end{array}\right]$ of $A$ is flat if $\operatorname{rank} A=\operatorname{rank} \tilde{A}$. Then a sample result is as follows: if $y=\left(y_{w}\right)_{\leqslant 2 k}$ is a truncated tracial sequence, $M_{k}(y)$ is positive semidefinite and flat over $M_{k-1}(y)$, then $y$ is a truncated tracial moment sequence [BK, Corollary 3.19].

In a different vein, the classical theorem of Hilbert was used by Fialkow and Nie to solve the bivariate quartic truncated moment problem [FN], Theorem 3.3]. The well-known duality between positive polynomials and the moment problem extends to the tracial noncommutative setting. That is, all truncated tracial sequences $\left(y_{w}\right)_{\leqslant 2 k}$ with positive definite tracial moment matrix $M_{k}(y)$ have a tracial moment representation if and only if all trace-positive polynomials of degree $\leqslant 2 k$ are elements of $\Theta^{2}$ [BK, Theorem 4.4]. Combining this with Theorem 3.1 yields the following solution to the bivariate quartic truncated tracial moment problem:

Corollary 3.8. Let $y=\left(y_{w}\right)_{\leqslant 4}$ be a bivariate truncated tracial sequence with a positive definite tracial moment matrix. Then $y$ is a tracial moment sequence.

As in the proof of [BCKP, Theorem 4.5], Carathéodory's theorem can be used to obtain bounds for the representation (3) of the sequence $y$ in Corollary 3.8 . That is, there are 15 pairs of symmetric $2 \times 2$ matrices $\left(A_{i}, B_{i}\right) \in\left(\mathbb{S R}^{2 \times 2}\right)^{2}$ and $\lambda_{i} \in \mathbb{R}_{\geqslant 0}$ which sum up to one, satisfying

$$
y_{w}=\sum_{i=1}^{15} \lambda_{i} \operatorname{tr}\left(w\left(A_{i}, B_{i}\right)\right) .
$$

\section{References}

[Akh] N.I. Akhiezer, The classical moment problem and some related questions in analysis, Hafner Publishing Co., 1965

[BT] C. Bayer and J. Teichmann, The proof of Tchakaloff's theorem, Proc. Amer. Math. Soc. 134, no. 10, 3035-3040, 2006

[BCKP] S. Burgdorf, K. Cafuta, I. Klep, and J. Povh, Semidefinite programming certificates for tracial matrix inequalities, preprint, http://www.optimization-online.org/DB_HTML/2010/04/2595.html

[BK] S. Burgdorf and I. Klep, The truncated tracial moment problem, accepted for publication in J. Operator Theory, http://arxiv.org/abs/1001.3679

[Con] A. Connes, Classification of injective factors. Cases $I I_{1}, I I_{\infty}, I I I_{\lambda}, \lambda \neq 1$, Ann. Math. 104, no. 1, 73-115, 1976

$[\mathrm{CF}]$ R.E. Curto and L.A. Fialkow, Solution of the truncated complex moment problem for flat data, Mem. Amer. Math. Soc. 119 , no. 568,1996

[CLR] M. D. Choi, T. Y. Lam, and B. Reznick, Extremal positive semidefinite forms, Math. Ann. 231, no. 1, 1-18, 1977/78

[FN] L. Fialkow and J. Nie, Positivity of Riesz functionals and solutions of quadratic and quartic moment problems, J. Funct. Anal. 258, no. 1, 328-356, 2010

[Hel] J.W. Helton, "Positive" non-commutative polynomials are sums of squares, Ann. of Math. (2) 156, no. 2, 675-694, 2002

[Hil] D. Hilbert, Über die Darstellung definiter Formen als Summe von Formenquadraten, Math. Ann. 32, 342-50, 1888

[KS] I. Klep and M. Schweighofer, Connes' embedding conjecture and sums of hermitian squares, Adv. Math. 217, no. 4, $1816-1837,2008$

[KN] M.G. Krein and A.A. Nudel'man, The Markov moment problem and extremal problems, Translations of Mathematical Monographs, 50, Amer. Math. Soc., 1977

[Mar] M. Marshall, Positive polynomials and sums of squares, Mathematical Surveys and Monographs, 146. Amer. Math. Soc., 2008

[McC] S. McCullough, Factorization of operator-valued polynomials in several non-commuting variables, Linear Algebra Appl. 326, no. 1-3, 193-203, 2001

[PD] A. Prestel and C.N. Delzell, Positive polynomials. From Hilbert's 17th problem to real algebra, Springer Monogr. Math., 2001

[PR] V. Powers and B. Reznick, Notes towards a constructive proof of Hilbert's theorem on ternary quartics, Quadratic forms and their applications (Dublin, 1999), 209-227, Contemp. Math., 272, Amer. Math. Soc., Providence, RI, 2000.

[PRSS] V. Powers, B. Reznick, C. Scheiderer, and F. Sottile, A new approach to Hilbert's theorem on ternary quartics, C. R. Math. Acad. Sci. Paris 339 (2004), no. 9, 617-620

[Put] M. Putinar, Positive polynomials on compact semi-algebraic sets, Indiana Univ. Math. J. 42, no. 3, 969-984, 1993

[Rez] B. Reznick, On Hilbert's construction of positive polynomials, preprint, http://arxiv.org/abs/0707.2156

[ST] J.A. Shohat and J.D. Tamarkin, The problem of moments, Amer. Mat. Soc. Surveys II, 1943 